\title{
苯甲醇的绿色选择性氧化
}

虞宗豪，洪宇轩，曹馨尹，孙宏枚”，赵蓓”，姚英明

苏州大学材料与化学化工学部, 江苏苏州 215123

摘要: 醇的氧化是制备醛和酮的重要反应之一, 也是有机化学理论教学和实验教学的一个重点内容。目前, 大多数 本科生实验教材安排了环己醇氧化合成环己酮的实验。虽然现有实验教材提供了一例将伯醇氧化为醛的方案, 即采 用 $\mathrm{Na}_{2} \mathrm{WO}_{4} \cdot 2 \mathrm{H}_{2} \mathrm{O} /\left(\mathrm{C}_{4} \mathrm{H}_{9}\right)_{4} \mathrm{NHSO}_{4}$ 催化体系, 在 $90{ }^{\circ} \mathrm{C}$ 用 $\mathrm{H}_{2} \mathrm{O}_{2}$ 将苯甲醇选择性氧化为苯甲醛, 但是存在着产率低、安 全性不高和重金属污染等问题。本文采用 $\mathrm{Fe}\left(\mathrm{NO}_{3}\right)_{3} \cdot 9 \mathrm{H}_{2} \mathrm{O}$ 、4-差基-2,2,6,6-四甲基哌啶氧化物(4-OH-TEMPO)和乙酸 ( $\mathrm{HOAc}$ )为催化剂、 $\mathrm{O}_{2}$ 为氧化剂, 以 $\mathrm{H}_{2} \mathrm{O}$ 为溶剂, 在温和的反应条件下高选择性地将苯甲醇氧化为苯甲醛, 分离产率 可达 $91 \%$, 这为伯醇的选择性氧化提供了一个低成本且高效绿色的实验教学新方案。同时, 在实验过程中引入薄层 色谱、气相色谱、快速制备液相色谱和核磁共振氢谱等手段对产物进行分离、提纯和表征, 有效提升了本科生的综 合实验技能和科研素养。

关键词: 伯醇; 铁(III)盐; 选择性氧化; 苯甲醛; 绿色化学

中图分类号: G64; O6

\section{Green and Selective Oxidation of Benzyl Alcohol}

\author{
Zonghao Yu, Yuxuan Hong, Xinyin Cao, Hongmei Sun *, Bei Zhao *, Yingming Yao \\ College of Chemistry, Chemical Engineering and Materials Science, Soochow University, Suzhou 215123, \\ Jiangsu Province, China.
}

\begin{abstract}
Oxidation of alcohols is an important method to produce aldehydes and ketones, and is an important component in organic chemistry theory and practical teaching. Currently, oxidation of cyclohexanol to cyclohexanone is commonly introduced in practical undergraduate textbooks. The only experiment designed for the preparation of aldehydes from primary alcohols in current practical textbooks is using the oxidant $\mathrm{H}_{2} \mathrm{O}_{2}$ for the selective oxidation of benzyl alcohol to benzaldehyde at $90{ }^{\circ} \mathrm{C}$ in the presence of a catalytic amount of $\mathrm{Na}_{2} \mathrm{WO}_{4} \cdot 2 \mathrm{H}_{2} \mathrm{O} /\left(\mathrm{C}_{4} \mathrm{H}_{9}\right)_{4} \mathrm{NHSO}_{4}$. However, this reaction has low-yields, potential safety issues, and concerns with environmental pollution. Here, we present a practical alternative reaction involving the mixed catalytic system of $\mathrm{Fe}\left(\mathrm{NO}_{3}\right)_{3} \cdot 9 \mathrm{H}_{2} \mathrm{O}, 4$-hydroxy-2,2,6,6tetramethyl-piperidinooxy (4-OH-TEMPO), and acetic acid (HOAc). This reaction allowed for a highly selective oxidation of benzyl alcohol to benzaldehyde with an isolated yield of up to $91 \%$ using $\mathrm{O}_{2}$ as the oxidant and $\mathrm{H}_{2} \mathrm{O}$ as the solvent under mild conditions. This new strategy for the selective oxidation of primary alcohols is a low-cost, and more importantly, highly efficient and green strategy. Additionally, several techniques such as thin layer chromatography, gas chromatography, flash preparative liquid chromatography, and ${ }^{1} \mathrm{H}$ NMR spectroscopy were used to detect and purify products, to improve experimental techniques, and to enhance the scientific literacy of undergraduate students.
\end{abstract}

Key Words: Primary alcohol; Fe(III) salt; Selective oxidation; Benzaldehyde; Green chemistry

收稿: 2021-10-18; 录用: 2021-11-04; 网络发表: 2022-01-28

“通讯作者, Emails: sunhm@suda.edu.cn (孙宏枚); zhaobei@suda.edu.cn (赵蓓)

基金资助: 江苏省高等学校自然科学研究重大项目(19KJA610001); 苏州大学第二批本科教学团队项目(5831501820); 化学国家一流本科专业建 设点专项经费 


\section{1 引言}

氧化反应是有机化学中的基本反应之一, 在有机合成中具有特殊重要的意义 ${ }^{[1]}$ 。选择性氧化是 有机化学理论教学中的一个重要内容, 其中, 伯醇的选择性氧化是一个典型例子。因为醛是一种用 途广泛的重要有机化学品, 但通过伯醇的氧化法制备时容易被直接氧化为羧酸。所以, 由伯醇制备 醛往往需要特殊试剂或反应条件来控制氧化进程 ${ }^{[2]}$ 。

苯甲醛是最简单的、也是工业上最常使用的芳香醛。目前, 面向本科生的有机化学实验教材中, 苯甲醇选择性氧化的实验方案是以 $\mathrm{Na}_{2} \mathrm{WO}_{4} \cdot 2 \mathrm{H}_{2} \mathrm{O} 、\left(\mathrm{C}_{4} \mathrm{H}_{9}\right)_{4} \mathrm{NHSO}_{4}$ 和 $30 \%$ 的 $\mathrm{H}_{2} \mathrm{O}_{2}$ 为催化-氧化体系, 在 $90^{\circ} \mathrm{C}$ 下反应, 再经减压蒸馏得到目标产物苯甲醛, 分离产率低(图 1, 方程式a) ${ }^{[3]}$ 。由于 $\mathrm{Na}_{2} \mathrm{WO}_{4} \cdot 2 \mathrm{H}_{2} \mathrm{O}$ 含有重金属、排放的废液具有环境污染性, 同时 $90{ }^{\circ} \mathrm{C}$ 下使用 $\mathrm{H}_{2} \mathrm{O}_{2}$ 存在安全隐患, 尤其是蒸馏时若有 残留的 $\mathrm{H}_{2} \mathrm{O}_{2}$ 可能引发爆炸。因此, 很有必要对这个实验进行改良。

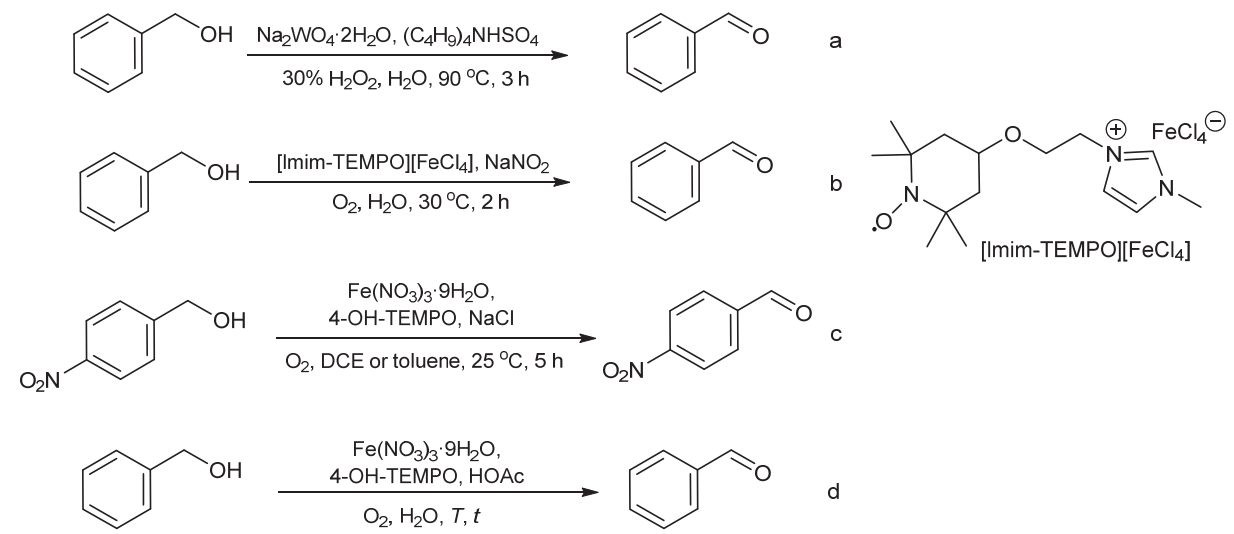

图1 苯甲醇选择性氧化为苯甲醛

通过文献调研, 我们发现伯醇的选择性氧化作为有机合成中一个非常重要的官能团转换, 受到 了广泛的关注。其中一个新进展就是基于铁系催化体系的 $\mathrm{O}_{2}$ 氧化法 ${ }^{[4-7]}$ 。铁是地壳中含量最丰富的金 属元素之一, 价格低廉, 同时具有很好的生物相容性, 是目前开发绿色金属催化剂的首选之一 ${ }^{[8]}$, 而 $\mathrm{O}_{2}$ 相对其他常用氧化剂而言, 也具有价廉、环境友好(如氧化副产物为水)等优势 ${ }^{[4]}$ 。因此, 基于铁系 催化体系的 $\mathrm{O}_{2}$ 氧化法为伯醇的选择性氧化提供了一个极具吸引力的新策略。2011年, 何良年和张锁 江等人 ${ }^{[9]}$ 设计了一个含 $\mathrm{Fe}^{3+}$ 离子的离子液体, 发现它与 $\mathrm{NaNO}_{2} 、 \mathrm{O}_{2}$ 组成的催化-氧化体系可以将苯甲 醇选择性地氧化为苯甲醛, 反应以 $\mathrm{H}_{2} \mathrm{O}$ 为溶剂, 但是使用毒性较强的 $\mathrm{NaNO}_{2}$ 限制了该方法的实用(图 1 , 方程式b)。2019年, 麻生明等人 ${ }^{[10]}$ 报道了以 $\mathrm{Fe}\left(\mathrm{NO}_{3}\right)_{3} \cdot 9 \mathrm{H}_{2} \mathrm{O}$ 、4-羟基-2,2,6,6-四甲基哌啶氧化物(4OH-TEMPO)、 $\mathrm{NaCl}$ 和 $\mathrm{O}_{2}$ 为催化-氧化体系, 实现了对硝基苯甲醇的选择性氧化(图1, 方程式c)。其中, 铁(III)盐和4-OH-TEMPO可以购买, 价格也较便宜, 但是该方案的优选溶剂是毒性较强的1, 2-二氯 乙烷(DCE)或者高沸点的甲苯, 遇 $\mathrm{H}_{2} \mathrm{O}$ 则不能顺利进行。于是, 我们希望能够综合上述两个研究成果 的优点, 以 $\mathrm{Fe}\left(\mathrm{NO}_{3}\right)_{3} \cdot 9 \mathrm{H}_{2} \mathrm{O}$ 和4-OH-TEMPO为催化剂、 $\mathrm{O}_{2}$ 为氧化剂、 $\mathrm{H}_{2} \mathrm{O}$ 为溶剂进行反应, 将苯甲醇 高选择性地氧化为苯甲醛, 获得适合本科生基础实验教学的新方案(图1, 方程式d)。

\section{2 实验部分}

\section{1 实验原理}

参考文献 ${ }^{[4,5]}$, 本实验的反应机理涉及两个催化循环过程。如图2所示, 在循环1中: 首先, 铁(III) 盐中的 $\mathrm{Fe}^{3+}$ 离子与 $4-\mathrm{OH}-\mathrm{TEMPO}$ 结合生成中间体 $\mathbf{1}$, 然后, 中间体 $\mathbf{1}$ 与苯甲醇反应, 释放 $\mathrm{H}^{+}$形成中间 体 2, 最后, 中间体 2 经过算氢反应生成苯甲醛, 同时生成 $\mathrm{Fe}^{2+}$ 离子和 $4-\mathrm{OH}-\mathrm{TEMPOH}$; 循环 2 则涉及 $\mathrm{Fe}^{2+}$ 离子和 $\mathrm{Fe}^{3+}$ 离子之间的转变, 其中 $\mathrm{O}_{2}$ 和 $\mathrm{NO}_{2}$ 起了关键的作用, 这个循环确保了循环 1 的顺利进行。 在催化剂预反应阶段, 逐渐产生的催化量 $\mathrm{NO}_{2}$ 来源于铁(III)盐 $\mathrm{Fe}\left(\mathrm{NO}_{3}\right)_{3} \cdot 9 \mathrm{H}_{2} \mathrm{O}$ 。 

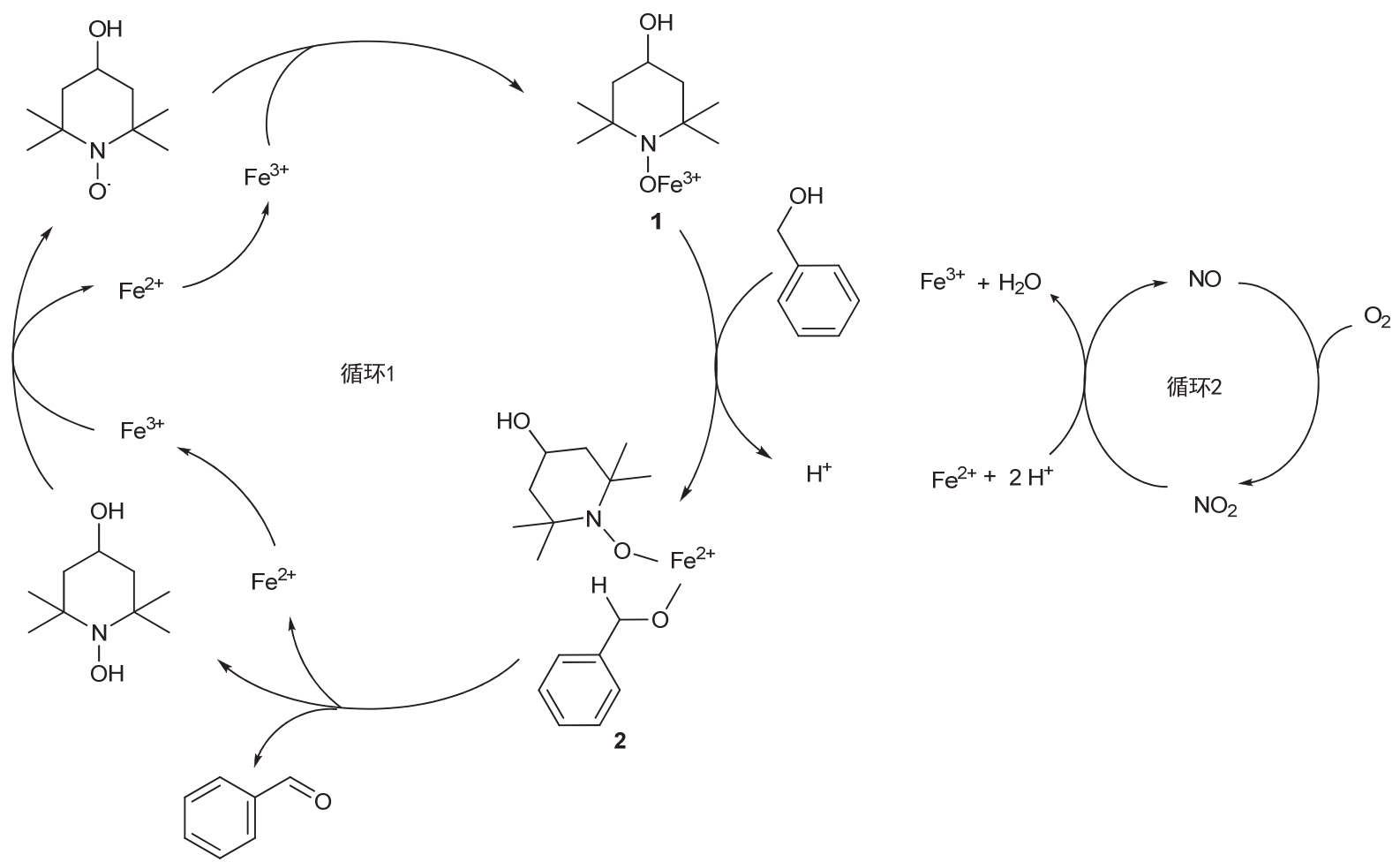

图2 本实验的反应机理

\section{2 试剂及材料}

实验试剂及材料如表1所示。

表1 实验试剂及材料

\begin{tabular}{|c|c|c|}
\hline 试剂名称 & 纯度 & 生产厂家 \\
\hline 九水合硝酸铁(III) & $98.5 \%$ & 上海麦克林生化科技有限公司 \\
\hline 乙酸 & 分析纯 & 国药集团化学试剂有限公司 \\
\hline 4-羟基-2,2,6,6-四甲基哌啶氧化物 & $\geq 99 \%$ & 上海笛柏生物科技有限公司 \\
\hline 苯甲醇 & 分析纯 & 国药集团化学试剂有限公司 \\
\hline 氯化钠 & 分析纯 & 国药集团化学试剂有限公司 \\
\hline 氯化铵 & 分析纯 & 江苏强盛功能化学股份有限公司 \\
\hline 乙酸铵 & 分析纯 & 上海强顺化学试剂有限公司 \\
\hline 氢氧化钠 & 分析纯 & 上海麦克林生化科技有限公司 \\
\hline 无水氯化钻 & 分析纯 & 国药集团化学试剂有限公司 \\
\hline 氧气 & 工业级 & 苏州天平晶元气体有限公司 \\
\hline 水 & 去离子水 & 苏州信致达科学仪器有限公司 \\
\hline 硅藻土 & $\geq 0.995$ & 生工生物工程(上海)股份有限公司 \\
\hline 柱层析硅胶(300-400目) & 无 & 青岛海洋化工厂分厂 \\
\hline 石油醚 $\left(60-90{ }^{\circ} \mathrm{C}\right)$ & 分析纯 & 江苏强盛功能化学股份有限公司 \\
\hline 乙酸乙酯 & 分析纯 & 江苏强盛功能化学股份有限公司 \\
\hline 正十二烷 & $\geq 99 \%$ & 上海笛柏生物科技 \\
\hline 氛代氯仿 & 99.8 原子 $\% \mathrm{D}(0.03 \% v / v \mathrm{TMS})$ & 北京百灵威科技有限公司 \\
\hline
\end{tabular}




\section{3 仪器和表征方法}

\subsection{1 实验仪器}

实验仪器如表2所示。

表2 实验仪器

\begin{tabular}{ccc}
\hline 仪器名称 & 型号 & 生产厂家 \\
\hline 气相色谱仪 & Agilent 8860 GC System & 安捷伦科技(中国)有限公司 \\
快速制备液相色谱仪 & SepaBean ${ }^{\circledR}$ machine U100 & 三泰科技(常州)有限公司 \\
核磁共振波谱仪 & AVANCE III HD 300 MHz & 布鲁克(北京)科技有限公司 \\
\hline
\end{tabular}

\subsection{2 表征方法}

(1) 气相色谱 $(\mathrm{GC})$ : 以乙酸乙酯为溶剂, $n-\mathrm{C}_{12} \mathrm{H}_{26}$ 为内标。色谱柱型号: $\mathrm{P} / \mathrm{N}: 19091 \mathrm{~J}-413(\mathrm{HP}-5$ $30 \mathrm{~m} \times 320 \mu \mathrm{m} \times 0.25 \mu \mathrm{m})$ 。升温程序: 初温 $80^{\circ} \mathrm{C}$ 保持 $1 \mathrm{~min}$, 程序升温速率 $20^{\circ} \mathrm{C} \cdot \mathrm{min}^{-1}$, 终温 $300^{\circ} \mathrm{C}$ 保持 $2 \mathrm{~min}$ 。运行后在 $280^{\circ} \mathrm{C}$ 保持 $2 \mathrm{~min}$ 。

(2) 快速制备液相色谱: 洗脱剂为 $V$ (石油醚): $V($ 乙酸乙酯 $)=9: 1$, 安装分离柱并润洗; 安装上样 柱，启动快速制备程序，用试管自动接收洗脱液。

(3) 核磁共振氢谱 $\left({ }^{1} \mathrm{H} N \mathrm{NMR}\right)$ : 将样品溶于含有 $0.03 \%$ (体积分数) TMS的氞代氯仿 $\left(\mathrm{CDCl}_{3}\right)$ 中，转 移到核磁管中充分摇匀, 室温下测定。

\section{4 实验步骤}

\subsection{1 预反应}

如图3所示搭建反应装置, 向 $50 \mathrm{~mL}$ 三颈圆底烧瓶中依次加入 $\mathrm{Fe}\left(\mathrm{NO}_{3}\right)_{3} \cdot 9 \mathrm{H}_{2} \mathrm{O}(0.56 \mathrm{~g}, 1.4 \mathrm{mmol})$ 、 5 滴乙酸 $(\mathrm{HOAc}$, 约 $0.25 \mathrm{~mL})$ 和4-OH-TEMPO $(0.48 \mathrm{~g}, 2.8 \mathrm{mmol})$, 塞上堵头, 打开弹簧夹, 通入 $\mathrm{O}_{2}$, 室温下搅拌预反应 $15 \mathrm{~min}$, 反应瓶内逐渐有红棕色气体出现。

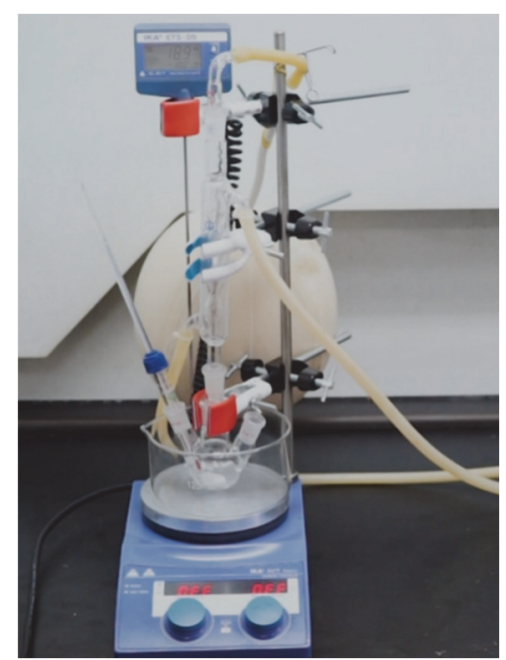

图3 反应装置示意图

\section{4 .2 苯甲醇的氧化}

用注射器分别量取 $20.0 \mathrm{~mL} \mathrm{H} \mathrm{H}_{2} \mathrm{O}$ 和 $2.1 \mathrm{~mL}(20 \mathrm{mmol})$ 苯甲醇, 依次从乳胶管处(图4红色圈注处)注 入。将水浴温度控制在 $50-55{ }^{\circ} \mathrm{C}$ 之间, 反应每 $1 \mathrm{~h}$ 用注射器取出极少量反应混合物, 通过薄层色谱法 (TLC)监控反应进程。TLC的展开剂为石油醚和乙酸乙酯的混合液, 体积比为 $5: 1$ 。约 $2 \mathrm{~h}$ 后反应趋于 完全, 停止加热和搅拌, 接上尾气吸收装置, 通过挤压气袋的方式排出尾气, 用 $\mathrm{NaOH}$ 溶液吸收。 


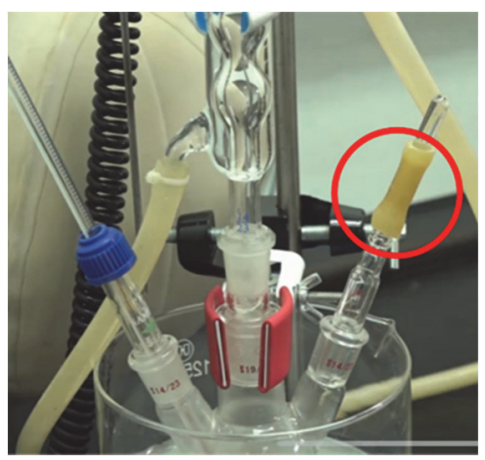

图4 红圈内为注射器加液体处

电子版为彩图

\subsection{3 苯甲醛的分离、提纯与表征}

待反应混合液冷却至室温后, 用硅藻土过滤, 再用 $20 \mathrm{~mL}$ 乙酸乙酯淋洗硅藻土。分液, 用 $5 \mathrm{~mL}$ 乙酸乙酯萃取水层一次。合并有机相, 加入适量无水 $\mathrm{CaCl}_{2}$ 干燥。过滤, 将滤液转移至圆底烧瓶中。 用旋转蒸发仪除去溶剂, 得到橙黄色粗产物。取极少量粗产物, 用乙酸乙酯稀释后进行 GC分析, 其 结果见图5。发现其中主要是目标产物苯甲醛。

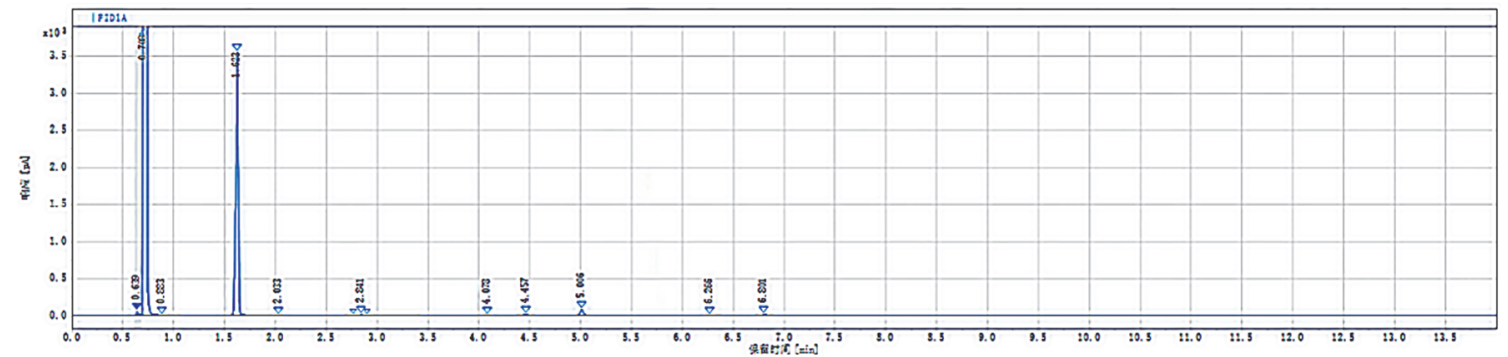

图5 反应混合物的 GC谱图

利用快速制备液相色谱仪对粗产物进行分离提纯, 根据检测曲线图(图6), 并结合TLC的检测结 果, 确定目标产物苯甲醛所在的接收管。合并目标洗脱液, 浓缩得到无色产品。通过减重法计算产 物的产量和产率。本实验可以得到 $1.91 \mathrm{~g}$ 苯甲醛, 分离产率为 $91 \%$ 。粗产物也可以通过柱色谱提纯, 并用TLC跟踪、检出。

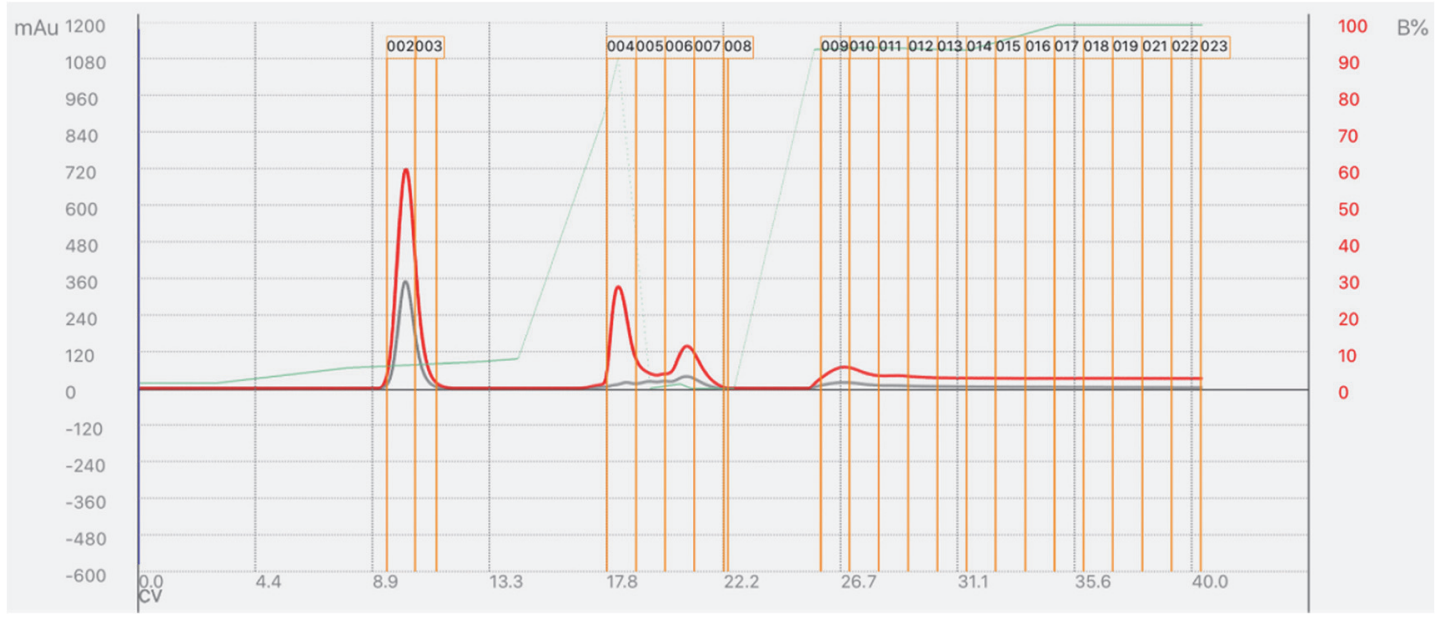

图6 粗产品的快速制备液相色谱仪的分离谱图 
所得产物用 ${ }^{1} \mathrm{H}$ NMR进行表征(图7), 确定为苯甲醛。实验到此结束, 历时约 $4-5 \mathrm{~h}$ 。
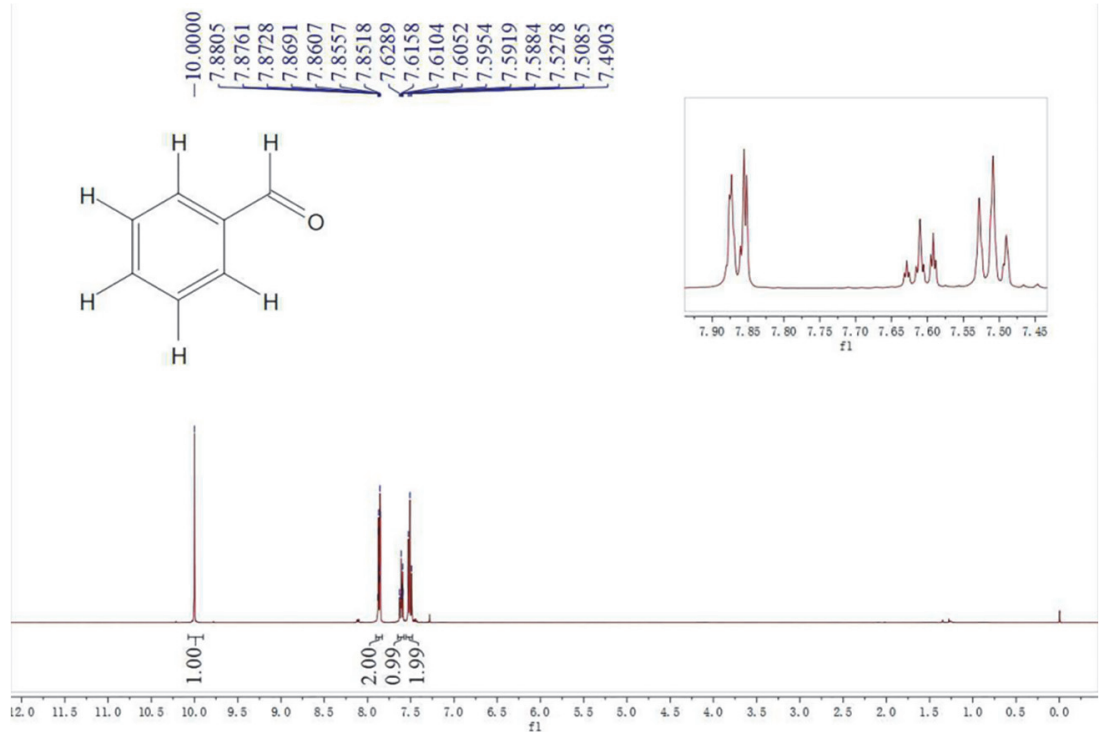

图7 产物苯甲酫的 ${ }^{1} \mathrm{H}$ NMR谱图(300 $\left.\mathrm{MHz}, \mathrm{CDCl}_{3}\right)$

\section{3 结果与讨论}

\section{1 实验条件篮选}

我们以水为溶剂, 对苯甲醇选择性氧化为苯甲醛进行了条件篎选, 结果见表 3 。首先, 我们参照 文献报道的反应条件, 即 $25^{\circ} \mathrm{C}$, 由 $\mathrm{Fe}\left(\mathrm{NO}_{3}\right)_{3} \cdot 9 \mathrm{H}_{2} \mathrm{O} 、 4-\mathrm{OH}-\mathrm{TEMPO} 、 \mathrm{NaCl}$ 和 $\mathrm{O}_{2}$ 组成催化-氧化体系 ${ }^{[10]}$, 仅将溶剂由原来的DCE改成 $\mathrm{H}_{2} \mathrm{O}$, 结果发现经过 $24 \mathrm{~h}$ 反应, 苯甲醛的 GC产率只有 $15 \%$ (表 3 , 序号 1 )。 考虑到 $\mathrm{HOAc}$ 既可能代替上述方案中 $\mathrm{NaCl}$ 的作用, 又能提供 $\mathrm{H}^{+}$促进 $\mathrm{Fe}^{2+}$ 离子和 $\mathrm{Fe}^{3+}$ 离子之间的转变 ${ }^{[10]}$, 我们以 HOAc代替 $\mathrm{NaCl}$ 进行实验, 果然发现苯甲醛的 $\mathrm{GC}$ 产率有所提升 (表 3 , 序号 2)。又考虑到 $\mathrm{NO}_{2}$ 在 此氧化反应中的作用, 我们增加了预反应环节, 希望通过提升 $\mathrm{NO}_{2}$ 的初始浓度来促进苯甲醛的生成。 结果确如我们所料, 在室温下经过 $15 \mathrm{~min}$ 预反应(最佳时间)后, 苯甲醇可以近乎 $100 \%$ 转化, 苯甲醛 的 $\mathrm{GC}$ 产率高达 $91 \%$ (表3, 序号3)。相比较, 添加 $\mathrm{NH}_{4} \mathrm{Cl}$ 和 $\mathrm{NH}_{4} \mathrm{OAc}$ 的效果较差(表3, 序号4和5)。随

表3 实验方案的优化过程 ${ }^{a}$

\begin{tabular}{|c|c|c|c|c|c|c|c|c|c|}
\hline $\begin{array}{l}\text { 实验 } \\
\text { 序号 }\end{array}$ & $\begin{array}{c}x(4-\mathrm{OH}- \\
\text { TEMPO)/\% }\end{array}$ & $\begin{array}{c}x\left(\mathrm{Fe}\left(\mathrm{NO}_{3}\right)_{3} .\right. \\
\left.9 \mathrm{H}_{2} \mathrm{O}\right) / \%\end{array}$ & $\begin{array}{c}x(\text { 添加物 }) / \\
\%\end{array}$ & $\begin{array}{c}V\left(\mathrm{H}_{2} \mathrm{O}\right) / \\
\mathrm{mL}\end{array}$ & $\begin{array}{c}\text { 水浴温度/ } \\
{ }^{\circ} \mathrm{C}\end{array}$ & $\begin{array}{c}\text { 反应时间/ } \\
h\end{array}$ & 预反应 & $\begin{array}{c}\text { 转化率/ } \\
\%\end{array}$ & $\begin{array}{c}\text { 产率/ } \\
\%\end{array}$ \\
\hline 1 & 10 & 5 & $\mathrm{NaCl}(10)$ & 6 & 25 & 24 & 否 & 39 & 15 \\
\hline 2 & 10 & 5 & HOAc (10) & 6 & 25 & 24 & 否 & 54 & 39 \\
\hline 3 & 10 & 5 & HOAc (10) & 6 & 25 & 24 & 是 & $>99$ & $91\left(20^{b}\right)$ \\
\hline 4 & 10 & 5 & $\mathrm{NH}_{4} \mathrm{Cl}(10)$ & 6 & 25 & 24 & 是 & 62 & 36 \\
\hline 5 & 10 & 5 & $\mathrm{NH}_{4} \mathrm{OAc}(10)$ & 6 & 25 & 24 & 是 & 76 & 50 \\
\hline 6 & 10 & 5 & HOAc (10) & 3 & 30 & 7 & 是 & $>99$ & 95 \\
\hline 7 & 10 & 5 & HOAc (10) & 3 & 50 & 2 & 是 & 78 & 69 \\
\hline 8 & 14 & 7 & HOAc (14) & 3 & 50 & 2 & 是 & $>99$ & $95\left(89^{c}\right)$ \\
\hline $9^{d}$ & 14 & 7 & HOAc (14) & 20 & 50 & 2 & 是 & $>99$ & $95\left(91^{c}\right)$ \\
\hline
\end{tabular}

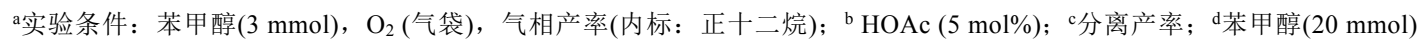


后, 我们将 $\mathrm{H}_{2} \mathrm{O}$ 的用量从 $6 \mathrm{~mL}$ 减少至 $3 \mathrm{~mL}$, 同时将水浴温度提高到 $30^{\circ} \mathrm{C}$, 通过 $\mathrm{GC}$ 检测发现反应可 以在 $7 \mathrm{~h}$ 内顺利完成(表 3 , 序号6)。考虑到本科生实验的课时要求, 我们进一步提高反应温度至 $50{ }^{\circ} \mathrm{C}$, 同时又稍微增加了催化剂的用量, 使得反应可以在 $2 \mathrm{~h}$ 内完成, 目标产物的分离产率可达 $89 \%$ (表 3 , 序号7和 8 )。考虑到本科生实验的可操作性, 也为了确保每一位本科生实验的重现性, 我们将苯甲醇 的用量由 $3 \mathrm{mmol}$ 增加至 $20 \mathrm{mmol}$, 发现反应在 $2 \mathrm{~h}$ 内也可以顺利完成(表 3 , 序号 9 ), 水浴温度在 $50-55^{\circ} \mathrm{C}$ 之间变化时, 目标产物的产率基本不变。

通过上述反应条件的篮选, 本实验的最佳方案是: $2.1 \mathrm{~mL}$ 苯甲醇 $(20 \mathrm{mmol}), 0.56 \mathrm{~g} \mathrm{Fe}\left(\mathrm{NO}_{3}\right)_{3} \cdot 9 \mathrm{H}_{2} \mathrm{O}$ $(x=7 \%), n\left(\mathrm{Fe}\left(\mathrm{NO}_{3}\right)_{3} \cdot 9 \mathrm{H}_{2} \mathrm{O}\right): n(4-\mathrm{OH}-\mathrm{TEMPO}): n(\mathrm{HOAc})=1: 1: 2$, 氧气袋, $20 \mathrm{~mL} \mathrm{H}_{2} \mathrm{O}$, 室温预反 应 $15 \mathrm{~min}$, 然后 $50-55^{\circ} \mathrm{C}$ 水浴反应 $2 \mathrm{~h}$, 可得到苯甲醛 $1.91 \mathrm{~g}$, 分离产率为 $91 \%$ 。

\section{2 产物分离与表征}

\subsection{1 粗产物表征}

反应混合物经硅藻土过滤、分液、干燥、浓缩后得橙黄色液态粗产物, 将该样品做了 GC检测(图 5)。为了方便比较, 我们还分别对苯甲醇、苯甲醛、苯甲酸和4-OH-TEMPO进行了 GC分析, 发现它 们的保留时间分别为 $2.033 、 1.641 、 3.016$ 和 $4.115 \mathrm{~min}$ 。在此基础上, 根据图 5 和薄层色谱的结果, 我 们发现粗产物中主要是目标产物苯甲醛(保留时间 $1.623 \mathrm{~min}$ ), 此外还包含4-OH-TEMPO和痕量的苯 甲醇，没有观察到苯甲酸。保留时间 $0.706 \mathrm{~min}$ 的信号归属于溶剂乙酸乙酯。

\section{2 .2 粗产物分离}

考虑到苯甲酫比较容易氧化且是无色液体, 因此我们采用快速制备液相色谱仪对粗产物进行分 离提纯, 结果如图6所示。

在正相柱色谱分离中, 极性较小的有机化合物会较快地被洗脱。因此, 根据上述分离谱图, 结 合TLC分析, 可以确定产物收集在 2 号和 3 号接收管中。我们合并 2 号、3号接收管中的洗脱液, 通过 旋转蒸发得到了目标产物。通过减重法计算出苯甲醛的产量是 $1.91 \mathrm{~g}$, 产率为 $91 \%$ 。

\subsection{3 产物的表征}

产物为无色液体, 其 ${ }^{1} \mathrm{H}$ NMR 谱图上有四组峰, $\delta$ 7.49-7.88的信号归属于苯环上的氢; $\delta 10.00$ 的 信号归属于甲酰基上的氢, 谱图中各峰面积之比为 $1: 2: 1: 2$ (图7), 由此可以确定所得产物为苯甲 醛。

\section{3 教学可行性}

在本实验方案中, 选用 $\mathrm{Fe}\left(\mathrm{NO}_{3}\right)_{3} \cdot 9 \mathrm{H}_{2} \mathrm{O} 、 4-\mathrm{OH}-\mathrm{TEMPO} 、 \mathrm{HOAc}$ 和 $\mathrm{O}_{2}$ 组成的催化-氧化体系, 避免 了已有实验教材中铇盐的重金属污染和过氧化氢的安全隐患。其中, $\mathrm{Fe}\left(\mathrm{NO}_{3}\right)_{3} \cdot 9 \mathrm{H}_{2} \mathrm{O} 、 4-\mathrm{OH}-\mathrm{TEMPO}$ 和HOAc可以直接购买使用, 价格也比较便宜, 使用 $\mathrm{H}_{2} \mathrm{O}$ 作为溶剂, 因此所需试剂成本低; $\mathrm{O}_{2}$ 可以直 接从氧气钢瓶用气袋接取, 实验全程不涉及明火操作, 安全性较高且清洁。反应过程中产生的 $\mathrm{NO}_{2}$ 是催化量的, 很容易通过 $\mathrm{NaOH}$ 溶液吸收处理。此外, 这一氧化反应对温度不是很敏感, 水浴温度在 50-55 ${ }^{\circ} \mathrm{C}$ 都可顺利进行, 便于操作。

反应过程中现象明显, 催化剂预反应时瓶内会逐渐出现红棕色气体, 当反应趋于完全时瓶内几 乎看不到红棕色, 同时溶液颜色变浅, 便于学生对反应进程的监测。

整个反应时间可控制在 $2 \mathrm{~h}$, 教师可以利用这段时间介绍气相色谱仪、快速制备液相色谱仪和核 磁共振波谱仪的使用和操作要领。总的实验时间约 $4-5 \mathrm{~h}$ 。

本实验设计中除了过滤、分液、干燥、萃取等常规操作以外, 还在分离、提纯和表征等过程中 引入了 TLC、GC、 ${ }^{1} \mathrm{H}$ NMR 和快速制备液相色谱等技术手段, 提升了本科生的实验技能, 拓宽了本 科生的专业视野, 与工业生产实际紧密联系, 符合现代化学实验教学对 “实验基本操作-小型综合实 验-综合实验”的多层次改革要求。

综合上述要点, 我们认为本次创新性实验设计适合推广至大规模的本科生基础实验教学。 


\section{4 结语}

本文报道了将苯甲醇高选择性氧化为苯甲醛的一个创新性改进实验方案。由于采用了绿色环保 且价廉的铁系催化剂、 $\mathrm{O}_{2}$ 为氧化剂, $\mathrm{H}_{2} \mathrm{O}$ 为溶剂, 使得反应绿色、安全性较高, 同时兼具高选择性和 良好的重现性。此外, 在实验过程中, 我们还采用了TLC和GC监测反应的进程, 使用快速制备液相 色谱仪和旋转蒸发仪对粗产物进行分离和提纯, 采用 ${ }^{1} \mathrm{H}$ NMR对目标产物进行表征。因此, 这样的创 新设计不仅满足了本科生基础实验的要求, 还提升了本科生的实验技能和专业素养, 与目前科学研 究和生产实际联系更加紧密。同时也将有助于培养本科生的绿色化学理念, 树立安全意识、环保意 识和社会责任感。

\section{参 考 文 献}

[1] 邢其毅, 裴伟伟, 徐瑞秋, 裴坚. 基础有机化学. 第4版. 北京: 北京大学出版社, 2017.

[2] Smith, M. B.; March, J. M. 高等有机化学一反应、机理与结构(原著第5版的修订版). 李艳梅, 译. 北京: 化学工业出版社, 2009.

[3] 高占先, 于丽梅. 有机化学实验. 第5版. 北京: 高等教育出版社, 2016.

[4] Wang, N. W.; Liu, R. H.; Chen, J. P.; Liang, X. M. Chem. Commun. 2005, 5322.

[5] Ma, S.; Liu J.; Li, S.; Chen, B.; Cheng, J.; Kuang, J.; Liu, Y.; Wan, B.; Wang, Y.; Ye, J.; et al. Adv. Synth. Catal. 2011, 353, 1005.

[6] Liu, J.; Ma, S. Org. Biomol. Chem. 2013, 11, 4186.

[7] Liu, J.; Ma, S. Org. Lett. 2013, 15, 5150.

[8] Bauer, I.; Knölker, H.-J. Chem. Rev. 2015, 115, 3170.

[9] Miao, C. X.; Wang, J. Q.; Bing, Y.; Cheng, W. G.; Sun, J.; Chanfreau, S.; He, L. N.; Zhang, S. J. Chem. Commun. 2011, $47,2697$.

[10] Jiang, X. G.; Liu, J. X.; Ma, S. M. Org. Process Res. Dev. 2019, 23, 825. 